\title{
A Prognostic Necroptosis-Related IncRNA Signature to Predict the Immune Response and Drug Sensitivity of Bladder Cancer
}

\section{Chao Tian}

District 2, Urology Department, the Yuebei People's Hospital, Shaoguan 512026, Guangdong, China.

\section{Guiliu Chen}

District 2, Urology Department, the Yuebei People's Hospital, Shaoguan 512026, Guangdong, China.

Zhengguo Cao (D19655975@qq.com)

District 2, Urology Department, the Yuebei People's Hospital, Shaoguan 512026, Guangdong, China.

\section{Research Article}

Keywords: necroptosis, IncRNA, bladder cancer, immune, drug

Posted Date: April 7th, 2022

DOI: https://doi.org/10.21203/rs.3.rs-1339521/v2

License: (c) (i) This work is licensed under a Creative Commons Attribution 4.0 International License.

Read Full License 


\section{Abstract}

Background: Patients with bladder cancer (BLCA), especially those with advanced stage cancer, have a short life span; thus, new drugs need to be developed urgently to improve the situation. Long noncoding RNAs (IncRNAs) are considered regulatory targets for cancer, including BLCA. Therefore, we constructed a promising signature of IncRNAs related to necroptosis and further predicted the immune response and sensitivity of drugs.

Methods: We downloaded the transcriptome and clinical data for BLCA from the Cancer Genome Atlas $\triangle T C G A$ D. Necroptosis related to IncRNAs (nrlncRNAs) was subsequently identified by univariate Cox regression. We then constructed the signature by performing the least absolute shrinkage and selection operator(LASSO) and evaluated it by Kaplan-Meier analysis, multivariate Cox regression, time-dependent receiver operating characteristics $(\mathrm{ROC})$, nomogram, calibration curves, gene set enrichment analyses(GSEA), immune checkpoint, tumor microenvironment(TME), N6-methyladenosine (m6A) modification genes, and the half-maximal inhibitory concentration (IC50) analysis in the risk groups.

Results: An 11-nrlncRNAs (AC008750.1, AL133297.1, AC005479.1, AP003559.1, UBE2Q1-AS1, MIR100HG, LINC02709, AC005387.1, LINC00649, AC021321.1, ETV7-AS1) prognostic signature was constructed. Immune checkpoint analysis revealed that the expression of most of the immune genes in the high-risk group was higher than those in the low-risk group. The signature predicted different IC50 values to have statistical significance in high-and low-risk groups for multiple drugs.

Conclusion: Our constructed signature may contribute to the prognosis of patients with BLCA as well as serve as clinical predictors of chemotherapy and immunotherapy.

\section{Introduction}

Bladder cancer (BLCA), with an estimated 550,000 new cases and 20,000 deaths in 2018, is one of the most common malignancies in the world, accounting for a large proportion of cancer deaths in men[1]. It can be broadly classified into nonmyometrial infiltrating BLCA (NMIBC) and muscle infiltrating BLCA (MIBC), with $25 \%$ of cases diagnosed as MIBC[2, 3]. The traditional treatment option for NMIBC is transurethral bladder tumor resection (TURBT). Radical cystectomy combined with chemotherapy is the primary treatment for MIBC[4]. Recently, immune checkpoint inhibitors, antibody-drug conjugates, and targeted therapies have gradually become options for certain patients[5]. Multiple trials have demonstrated that checkpoint inhibitors in advanced BLCA, including avelumab, atezolizumab, durvalumab, and nivolumab exert therapeutic effects by targeting the PD1/PD-L1 pathway. However, < $50 \%$ of the patients showed sensitivity to checkpoint inhibitors. It has been found that tumors seem to promote the formation of an immunosuppressive microenvironment via various mechanisms to resist immunotherapy[6]. Therefore, it is a new research direction to find gene targets for improving TME and promoting immunotherapy. 
Apoptosis has long been recognized as a powerful anticancer agent, but its resistance usually results in chemotherapy failure. Finding new nonapoptotic forms of programmed cell death (PCD) is imperative [7]. Necroptosis is also a type of PCD, and over the years, it has been shown to be triggered by a variety of stimuli, including Toll-like receptors(TLRs), death and interferon(IFN) receptor ligands, and certain pathogens[8]. Shikonin, a pyruvate kinase M2 (PKM2) inhibitor, can improve cisplatin resistance through BLCA cell necroptosis [9].Necroptosis is tightly regulated, requiring activation of the receptor-interacting protein (RIP) kinases RIPK1 and RIPK3. Necroptosis cells activated RIPK3 phosphorylates mixed lineage kinase domain-like protein(MLKL), which can oligomerize and translocate to the plasma membrane, resulting in the permeabilization of the necrotic plasma membrane and release of damage-related molecular patterns(DAMPs) that evoke the immune response[10]. Hence, necroptosis is referred to as an immunogenic cell death (ICD). It may be another beneficial factor in tumor clearance and/or malignancy restriction, depending on the stimulation of ICD in the TME[11]. Vaccinia virus (VV) can activate necroptosis and promote the induction of $T$ cell-mediated immune responses[12].

LncRNAs are RNA transcripts of $>200$ nucleotides in length and have secondary and tertiary structures. These allow them to perform functions similar to those of proteins[13]. LncRNAs play an essential role in cancer. Genome-wide expression patterns in tumor samples have identified a large number of IncRNAs associated with various types of cancer. LncRNA expression and its mutations may maintain or regulate tumor characteristics, such as proliferation and metastasis. LncRNAs have promising prospects as novel biomarkers and therapeutic tagets for cancer[14]. At present, the role of nrlncRNAs in BLCA cells has not been studied. This study aimed to provide a new therapeutic target for BLCA resistant to chemotherapy or immunotherapy.

\section{Materials And Methods}

\subsection{Patients and datasets}

To obtain a matrix of BLCA data, we downloaded clinical data and BLCA FPKM-standardized RNA transcriptome datasets (HTSeq-FPKM) from the TCGA website (https://portal.gdc.cancer.gov/): normal count, 19 and tumor count, 414. The RNA transcript data were arranged into gene symbols and each sample gene expression matrix using Strawberry Perl for further study. Futime, fustat, age, gender, grade, and stage (T, N, and M) for each sample were extracted from clinical data. Patients with BLCA with OS of $<30$ days were excluded from the study.

\subsection{Identification and correlation analysis of nrlncRNAs and network construction}

We identified 67 genes associated with necroptosis from previous reports[15] (Appendix Table 1A). First, we distinguished IncRNAs and mRNAs and explored the correlation between necroptosis gene and IncRNA expression. The "limma" R package (Pearson correlation coefficients $>0.4$ and $p$ value $<0.001$ ) was used to screen out highly correlated 1131 nrlncRNAs. Using the "igraph" R package, an interaction network was established to show the relationship between IncRNAs and necroptosis genes (NRGs). 
2.3 Analysis of differentially expressed nrlncRNAs and risk signature construction

We used log 2 fold change $(F C)>1$ and false discovery rate $(F D R)<0.05$ as screening criteria to obtain the differentially expressed nrlncRNAs. NrIncRNAs related to survival $(p<0.05)$ were screened by univariate Cox regression analysis based on downloaded clinical data. Next, we ran 1,000 cycles to obtain the model gene set through Lasso regression with a $p$ value of 0.05 , and 10 -fold cross-validation. A model was constructed after 1,000 random stimuli per cycle. The forest map was drawn using the result of the ggplot2 $\mathrm{R}$ package, and then the receiver operating characteristics (ROC) curves of the mode were plotted. We calculated the risk score for all samples using the following formula:

Risk score $=\sum{ }_{1}^{n}$ coef(nrlncRNAn) expr(nrlncRNAn).Coef(nrlncRNAn) represents the coefficient of nrlncRNAs that correlate with survival.Expr(nrlncRNAn) indicates the expression levels of nrIncRNAs. The BLCA samples were divided into high-and low-risk groups based on the median risk score. The different OS times between the two groups were analyzed using Kaplan-Meier plotter and $\mathrm{R}$ packet "survival."

\subsection{Independence factor analysis}

Univariate and multivariate Cox regression analyses were performed to assess whether risk score and clinical features were independent factors, and the different factors were compared by ROC analysis.

\subsection{Nomogram}

We used the rms R package to construct the nomogram including risk score, age, gender, and stage ( $T, N$, and $\mathrm{M}$ ) to predict the 1-, 3-, and 5-year OS rates of patients with BLCA. We used a calibration curve based on the Hosmer-Lemeshow test to examine whether the predicted outcome was consistent with the practical outcome.

2.6 Gene set enrichment analyses (GSEA)

GSEA was performed using the GSEA 4.2.1. Enriched pathways $(p<0.05$ and FDR $<0.25)$ were considered between the low- and high-risk groups.

\subsection{Immune, TME, and M6A analyses}

The immune cells and immune pathways were estimated using single-sample GSEA (sSGSEA). We downloaded the tumor immune cell infiltration file from TCGA. We subsequently calculated the sample's immune cell infiltration status including XCELL, TIMER, CIBERSORT, QUANTISEQ, MCPCOUNTER, EPIC, and CIBERSORT-ABS on TIMER2.0 (http://timer.cistrome.org/) and mapped the bubbles. We also compared immune checkpoint activation and TME scores between the low-risk and high-risk groups using ggplot2, ggpubr and estimated R packages. We collated $13 \mathrm{M} 6 \mathrm{~A}$ regulatory factors from previously published studies. (Appendix Table 1B). The m6A-related gene expression of high and low-risk groups were compared using the limma, reshape2, ggplot2, and ggpubr R packages. 


\subsection{Drug sensitivity}

Next, we used the pROphetic $\mathrm{R}$ package to assess drug sensitivity between high- and low-risk groups by comparing the half-maximal inhibitory concentration (IC50) of each patient with BLCA with Genomics of Drug Sensitivity in Cancer (https://www.cancerrxgene.org/)[16].

\subsection{Tumor cluster}

Potential clusters between tumor samples were explored using the ConsensusClusterPlus (CC) R package based on prognostic 11-IncRNA expression. Principal component analysis (PCA) was performed using the Rtsne R package. Differences in immune cell infiltration, immunocheckpoint, TME, and drug sensitivity analyses were compared among the BLCA sample clusters.

\section{Results}

\subsection{Identification of necroptosis-related IncRNAs in patients with BLCA}

The study workflow is illustrated in Fig. 1A. In this study, we used data from 433 patients with BLCA from the TCGA cohort $(T=414, N=19)$. After finding all IncRNAs and establishing coexpression analysis with 67 NRGs to identify IncRNAs related to necroptosis (coefficients $>0.4$ and $p<0.001$ ), we established an interactive network to demonstrate the relationship between nrlncRNAs and NRGs (Fig. 1B). Next, 686 nrlncRNAs with significant differences were identified by differential expression analysis (FC $>1$ and FDR $<0.05$ ). We found that $108 \mathrm{nrlncRNAs}$ were downregulated, while the others were upregulated, mapping the volcano plot (Fig. 1C). We selected up-and downregulated nrlncRNAs of the top 50 most significant differences in the heat map (Fig. 1D).

\subsection{Construction of prognostic necroptosis-related IncRNAs signature}

According to univariate Cox regression analysis, nrlncRNAs related to survival $(p<0.05)$ were screened and plotted on forest map (Fig. 1E). The Sankey diagram illustrates the regulatory relationship between these IncRNAs and NRGs (such as TSC1, BRAF, and ATRX) (Fig. 1F). To avoid over-fitting of the signature, when the first value of $\log (\lambda)$ was the minimum possible deviance, we performed LASSO regression and multiple Cox regression analysis on these nrlncRNAs (Fig. 1G and H). Finally, an 11-necroptosis-related IncRNA signature was established.

Risk score was calculated using the following formula: Risk score $=($ AC008750.1 $\times$ $-2.18520147058094)+(A L 133297.1 \times 2.05280592077303)+\square A C 005479.1 \times 1.35382239745128 \rrbracket+$ $($ AP003559.1 $\times 1.28533807015561)+($ UBE2Q1-AS1 $\times-1.16093696050806)+($ MIR100HG $\times$ $0.449749505941033)+($ LINC02709 $\times 1.25314467417777)+($ AC005387.1 $\times-0.607382896530388)+$ $($ LINC00649 $\times-0.490052688800663)+($ AC021321.1 $\times-1.16608863558295)+($ ETV7-AS1 $\times$ $-0.589464760152836)$ (Appendix Table 2). Patients were further divided into high- and low-risk groups, with the median risk score as the standard. When the model was constructed, we divided the samples 
into two groups (train and test) and obtained OS analysis, risk score curve, survival status, and 11nrlncRNAs risk heat map (Fig. 2A-C).

Univariate and multivariate Cox regression analyses were used to compare the risk scores of the signature with age, gender, grade, and stage (Fig. 3A and B). Area under the ROC curve (AUC) of 1-, 3-, and 5 -years survival were $0.736,0.735$, and 0.744 , respectively, and AUCs of the risk score $(0.736)$ compared with other factors including age (0.662), gender(0.479), grade (0.529), and stage $(0.641)$, respectively (Fig. 3C and D).

\subsection{Nomogram}

We divided patients with BLCA into stage I/II and stage III/IV groups, and then compared whether OS (survival probability) of patients in high-or low-risk groups had significant differences in the two stages according to the risk score of the model. We built a nomogram for predicting the 1-, 3-, and 5-year survival rates of patients with BLCA and plotted the 1-, 3-, and 5-year calibration curves (Fig. 3E-I).

\subsection{GSEA}

To investigate the functional and enrichment pathway differences between high-and low-risk groups, we used GSEA software to explore the Kyoto Encyclopedia of Genes and Genomes pathway throughout the entire set. The high-and low-risk groups enriched the first five pathways, and the BLCA pathway was built using multiple GSEA. The BLCA pathway $(p 0.01<0.05$; FDR $0.044<0.25$; |NES| $1.78>1.5)$ was significant in the risk group of this model (Fig. 4A and B) (Appendix Fig. 1A)

\subsection{Immunity factors, TME, and M6A analyses in risk groups}

The relationship between immune cells and the patient's risk score is depicted in the immune cell bubble chart. Cancer-associated fibroblast_XCELL, endothelial cell_MCP-counter, granulocyte-monocyte progenitor XCELL, macrophage M1_QUANTISEQ, macrophage M2_CIBERSORT, etc. were positively correlated with BLCA patients'risk scores $(p<0.05)$. However, B cell memory CIBERSORT, B cell_TIMER, T cell CD4 + central memory_XCELL, T cell CD8 + naive_XCELL, and regulatory T cells (Tregs)_CIBERSORTABS, among others, showed a negative correlation $(p<0.05)$ (Fig. 4C). Specific correlation coefficients and $p$-values for each type of immune cell are shown in Appendix Fig. 1B and Appendix Table 1C. SSGSEA showed that there were significant differences in immune cells such as macrophages, mast cells, neutrophils, Th1-cells, and Treg and immune-related function scores such as APC-co-inhibition and APCco-stimulation between high- and low-risk groups, respectively $(p<0.001)$ (Fig. 4D and E). Stromal cells, immune cells, and composite scores of each BLCA sample are shown, and the differences in these scores were compared between the high- and low-risk groups $(p<0.05)(\mathrm{Fig} .4 \mathrm{~F}-\mathrm{H})$. We found higher immune checkpoint gene expression in the high-risk group. There were significant differences in the expression of many genes, such as CD274, CD44, CD276, and HAVCR2, between the high-and low-risk groups (Fig. 4I). Significant differences were found in multiple m6A regulators gene expression in risk groups such as FTO, ALKBH5, METTL3, and YTHDC1 (Fig. 4G). 


\subsection{Distinguishing BLCA clusters and cluster evaluation}

We performed nine cluster comparisons of BLCA samples and found the weakest correlation between the two subtypes. We divided the BLCA samples into two distinct subtypes (C1 and C2) (Appendix Fig. 2). There are usually differences in immunotherapy and survival analysis between the $\mathrm{C} 1$ and $\mathrm{C} 2$ subtypes[17] (Fig. 5A). We observed a significant difference in survival probability between $\mathrm{C} 1$ and C2 ( $p$ $=0.032)($ Fig. $5 B$ ). In addition, we used the ggalluvial graph to show the relationship between high/low risk groups and C 1/C 2 clusters (Fig. 5C). PCA analysis showed that the expression of IncRNAs in BLCA could be used to scatter the sample clusters (C1 and C2) (Fig. 5D and E).

The differences in stromal cells, immune cells, and composite scores in each BLCA sample were compared between the $\mathrm{C} 1$ and $\mathrm{C} 2$ groups $(p<0.05)(\mathrm{Fig} .5 \mathrm{~F}-\mathrm{H})$. Heat map revealed differences among different immune cells in the tumor clusters. T cell CD4+_TIMER, T cell CD8+_TIMER, and Neutrophil_TIMER immune cells were highly concentrated in the $\mathrm{C} 1$ cluster (Fig. 8I). Immune checkpoint gene expressions such as TNFRSB4, CD274, and CD70 were mostly higher in the C1 cluster than in the C2 cluster (Fig. 5J).

\subsection{Drug sensitivity}

The IC50 of many chemotherapeutic or targeted drugs showed significant differences between the highand low-risk groups of the prognostic signature. This also suggests that the use of this model to differentiate patients can improve drug sensitivity to some extent and avoid drug resistance, especially some commonly used chemotherapeutic or targeted drugs such as cisplatin, dasatinib, docetaxel, gefitinib, imatinib, nilotinib, methotrexate, and lenalidomide (Appendix Fig. 3A). The same pattern was predicted for the $\mathrm{C} 1$ and $\mathrm{C} 2$ clusters (Appendix Fig. 3B).

\section{Discussion}

Cancer chemotherapy can no longer depend on a single drug treatment, such as cisplatin, as one chemotherapy drug cannot work for all patients and be 100\% effective against cancer progression. Resistance to anticancer drugs is caused by a number of factors, including individual differences that make resistance to chemotherapy more common[18]. Clinically, the survival rates for patients with advanced or metastatic BLCA remain low[19]. Thus, more attention should be paid to individualized treatment of patients. The establishment of a prognostic signature plays a key role. We developed an 11nrlncRNAs prognostic signature that could classify the BLCA samples into high- and low-risk groups and further evaluate the differences between the two groups, including survival analysis $(p<0.05)$, immunity factors, TME, and M6A analysis. We hope that this model will provide a new treatment strategy for BLCA.

The inhibitory role of necroptosis in cancer has been extensively studied. However, very few cases of BLCA have been reviewed. The PKM2 inhibitor shikonin, a PKM2 inhibitor, can overcome cisplatin resistance and promote necroptosis[9]. ABT 737, a bcl-2 family inhibitor, can also induce necroptosis in BLCA cells[20]. There may also be crosstalk between necroptosis and other forms of PCD, such as 
ferroptosis or pyroptosis[21]. Researchers constructed a 9-ferroptosis-related IncRNAs model to predict the prognosis of patients with BLCA and found that they were more sensitive to anti-PD-1/L1 immunotherapy and chemotherapy drugs, such as sunitinib, paclitaxel, docetaxel, and docetaxel[22]. Recently, an 8-nrlncRNAs prognostic signature associated with pyroptosis was developed to explore the molecular markers of BLCA[23]. We recently constructed a model of necroptosis, according to which the samples were scored and divided into high-and low-risk groups. High-risk patients were found to be more sensitive to a variety of drugs such as cisplatin, dasatinib, docetaxel, and imatinib. It also provides a direction for our clinical treatment to rely on this model. In addition, we also found that diverse drugs have different sensitivities to tumor clusters.

Immunotherapy has been widely used in clinical and scientific research in recent years. The main benefit of immune checkpoint inhibitor-related drugs over conventional chemotherapy is that patients with BLCA are more resistant to them and lead to a reduction in adverse events. Unfortunately, the response rate to immune checkpoint inhibitor is relatively low[24]. Immune function scores including APC_co_inhibition, APC_CO_stimulation, CCR, checkpoint, T_cell_ co_stimulation, and parainflammation were significantly different between the high-and low-risk groups. Thus, we can explore which immune checkpoints are more beneficial to the patient based on the patient's classification. CD274, CD44, HAVCR2, PDCD1LG2, LAIR1, CD276, TNFSF9, TNFRSF8, CD86, TNFRSF4, NRP1, and CD80 were highly expressed in the highrisk group. CD274 (PD-L1 promoter gene) methylation is an independent prognostic factor for BLCA[25]. CD44 is a surface marker of cancer stem cells and has also been found to be associated with drug resistance to BLCA treatment[26]. Conversely, TNFRSF14, TNFRSF25, CD160, LGALS9, andTMIGD2 were highly expressed in the low-risk group. The increased expression of TNFRSF14 can inhibit the growth of BLCA cells[27]. This suggests that our prognostic model is useful for BLCA classification and individualized treatment. Examining the immune checkpoint sensitivity of different BLCA samples may improve the response rate to immunotherapy.

$\mathrm{M} 6 \mathrm{~A}$ is the most common methylation modification and plays an essential role in IncRNA expression stability[28]. In this study, m6A regulator (FTO, ALKBH5) gene expression was higher in the high-risk group $(p<0.001)$. The expression of METTL3 and YTHDC1 was higher in the low-risk group $(p<0.001)$. FTO can promote BLCA growth by regulating the MALAT/miR-384/MAL2 axis by M6A RNA modification[29]. ALKBH5 has been shown to inhibit BLCA cell proliferation and sensitize cells to cisplatin by m6A-casein kinase 2 (CK2)a[30]. Therefore, the role of m6A methylase is more dependent on downstream genes in BLCA.

The limitations of this study include the lack of experimental evidence for the role of 11-nrlncRNAs (AC008750.1, AL133297.1, AC005479.1, AP003559.1, UBE2Q1-AS1, MIR100HG, LINC02709, AC005387.1, LINC00649, AC021321.1, and ETV7-AS1) in BLCA. LINC00649 has been found to promote the malignant progression of BLCA cells via the miR-15a-5p/HMGA1[31] and miR-16-5p/JARID2[32] axes. The roles of other nrlncRNAs still need to be explored further in the future.

\section{Conclusions}


In conclusion, our constructed signature may contribute to the prognosis of patients with BLCA as well as serve as clinical predictors of chemotherapy and immunotherapy.

\section{Abbreviations}

IncRNA

long non-coding RNA

BLCA

bladder cancer

TCGA

the Cancer Genome Atlas

nrlncRNAs

necroptosis related to IncRNAs

LASSO

least absolute shrinkage and selection operator

ROC

receiver operating characteristics

GSEA

gene set enrichment analyses

$\mathrm{m} 6 \mathrm{~A}$

N6-methyladenosine

IC50

the half-maximal inhibitory concentration

PCA

principal component analysis

OS

overall survival

NMIBC

non-myometrial infiltrating bladder cancer

MIBC

muscle infiltrating bladder cancer

TURBT

transurethral bladder tumor resection

TME

tumor microenvironment

PCD

programmed cell death

TLRs

Toll-like receptors

IFN 
interferon

PKM2

pyruvate Kinase M2

RIP

receptor-interacting protein

MLKL

mixed lineage kinase domain-like protein

DAMPs

damage-related Molecular Patterns

ICD

immunogenic cell death

sSGSEA

single-sample gene set enrichment analysis

GDSC

Genomics of Drug Sensitivity in Cancer

CC

ConsensusClusterPlus

NRGs

necroptosis genes

AUC

Area Under ROC Curve

CK2

casein kinase 2

\section{Declarations}

\section{Competing interests}

The authors declare that the research was conducted in the absence of any commercial or financial relationships that could be construed as a potential conflict of interest.

\section{Author Contributions:}

CT and GC wrote the first draft of the manuscript. ZC designed and modified this study. All authors contributed to the article and approved the submitted version.

\section{Data Availability}

The data supported the results are available at the TCGA (https://tcga-data.nci.nih.gov/tcga/) and GDSC (https://www.cancerrxgene.org/). The original contribution can be directed to the corresponding author in the study. 


\section{Funding}

Not applicable

\section{Acknowledgements}

Not applicable

Consent for publication

\section{References}

1. Bray, F., et al., Global cancer statistics 2018: GLOBOCAN estimates of incidence and mortality worldwide for 36 cancers in 185 countries. CA Cancer J Clin, 2018. 68(6): p. 394-424.

2. Dobruch, J., et al., Gender and Bladder Cancer: A Collaborative Review of Etiology, Biology, and Outcomes. Eur Urol, 2016. 69(2): p. 300-10.

3. Cumberbatch, M.G.K., et al., Epidemiology of Bladder Cancer: A Systematic Review and Contemporary Update of Risk Factors in 2018. Eur Urol, 2018. 74(6): p. 784-795.

4. DeGeorge, K.C., H.R. Holt, and S.C. Hodges, Bladder Cancer: Diagnosis and Treatment. Am Fam Physician, 2017. 96(8): p. 507-514.

5. Lenis, A.T., et al., Bladder Cancer: A Review. Jama, 2020. 324(19): p. 1980-1991.

6. Crispen, P.L. and S. Kusmartsev, Mechanisms of immune evasion in bladder cancer. Cancer Immunol Immunother, 2020. 69(1): p. 3-14.

7. Gong, Y., et al., The role of necroptosis in cancer biology and therapy. Mol Cancer, 2019. 18(1): $\mathrm{p}$. 100.

8. He, S., et al., Toll-like receptors activate programmed necrosis in macrophages through a receptorinteracting kinase-3-mediated pathway. Proc Natl Acad Sci U S A, 2011. 108(50): p. 20054-9.

9. Wang, Y., et al., PKM2 Inhibitor Shikonin Overcomes the Cisplatin Resistance in Bladder Cancer by Inducing Necroptosis. Int J Biol Sci, 2018. 14(13): p. 1883-1891.

10. Zhu, F., et al., Complex roles of necroptosis in cancer. J Zhejiang Univ Sci B, 2019. 20(5): p. 399413.

11. Scarpitta, A., et al., Pyroptotic and Necroptotic Cell Death in the Tumor Microenvironment and Their Potential to Stimulate Anti-Tumor Immune Responses. Front Oncol, 2021. 11: p. 731598. 
12. Ma, J., et al., Characterization of virus-mediated immunogenic cancer cell death and the consequences for oncolytic virus-based immunotherapy of cancer. Cell Death Dis, 2020. 11(1): p. 48.

13. Novikova, I.V., S.P. Hennelly, and K.Y. Sanbonmatsu, Tackling structures of long noncoding RNAs. Int J Mol Sci, 2013. 14(12): p. 23672-84.

14. Bhan, A., M. Soleimani, and S.S. Mandal, Long Noncoding RNA and Cancer: A New Paradigm. Cancer Res, 2017. 77(15): p. 3965-3981.

15. Zhao, Z., et al., Necroptosis-Related IncRNAs: Predicting Prognosis and the Distinction between the Cold and Hot Tumors in Gastric Cancer. J Oncol, 2021. 2021: p. 6718443.

16. Geeleher, P., N.J. Cox, and R.S. Huang, Clinical drug response can be predicted using baseline gene expression levels and in vitro drug sensitivity in cell lines. Genome Biol, 2014. 15(3): p. R47.

17. Das, S., K. Camphausen, and U. Shankavaram, Cancer-Specific Immune Prognostic Signature in Solid Tumors and Its Relation to Immune Checkpoint Therapies. Cancers (Basel), 2020. 12(9).

18. Gottesman, M.M., Mechanisms of cancer drug resistance. Annu Rev Med, 2002. 53: p. 615-27.

19. Bellmunt, J., Bladder cancer. Hematol Oncol Clin North Am, 2015. 29(2): p. xiii-xiv.

20. Cheng, R., et al., ABT-737, a Bcl-2 family inhibitor, has a synergistic effect with apoptosis by inducing urothelial carcinoma cell necroptosis. Mol Med Rep, 2021. 23(6).

21. Tang, R., et al., Ferroptosis, necroptosis, and pyroptosis in anticancer immunity. J Hematol Oncol, 2020. 13(1): p. 110.

22. Chen, M., et al., A New Ferroptosis-Related IncRNA Signature Predicts the Prognosis of Bladder Cancer Patients. Front Cell Dev Biol, 2021. 9: p. 699804.

23. Lia, T., et al., Development and validation of Pyroptosis-related IncRNAs prediction model for bladder cancer. Biosci Rep, 2022.

24. Bednova, O. and J.V. Leyton, Targeted Molecular Therapeutics for Bladder Cancer-A New Option beyond the Mixed Fortunes of Immune Checkpoint Inhibitors? Int J Mol Sci, 2020. 21(19).

25. Xu, J., et al., CD274 (PD-L 1) Methylation is an Independent Predictor for Bladder Cancer Patients' Survival. Cancer Invest, 2022: p. 1-6.

26. Wu, C.T., et al., Impact of CD44 expression on radiation response for bladder cancer. J Cancer, 2017. 8(7): p. 1137-1144.

27. Zhu, Y.D. and M.Y. Lu, Increased expression of TNFRSF14 indicates good prognosis and inhibits bladder cancer proliferation by promoting apoptosis. Mol Med Rep, 2018. 18(3): p. 3403-3410. 
28. Liu, H., et al., A novel N6-methyladenosine (m6A)-dependent fate decision for the IncRNA THOR. Cell Death Dis, 2020. 11(8): p. 613.

29. Tao, L., et al., FTO modifies the m6A level of MALAT and promotes bladder cancer progression. Clin Transl Med, 2021. 11(2): p. e310.

30. Yu, H., et al., ALKBH5 Inhibited Cell Proliferation and Sensitized Bladder Cancer Cells to Cisplatin by m6A-CK2a-Mediated Glycolysis. Mol Ther Nucleic Acids, 2021. 23: p. 27-41.

31. Chen, $\mathrm{X}$. and S. Chen, LINC00649 promotes bladder cancer malignant progression by regulating the miR-15a-5p/HMGA1 axis. Oncol Rep, 2021. 45(4).

32. Liu, Y., et al., LINC00649 Facilitates the Cellular Process of Bladder Cancer Cells via Signaling Axis miR-16-5p/JARID2. Urol Int, 2021: p. 1-9.

\section{Figures}

A

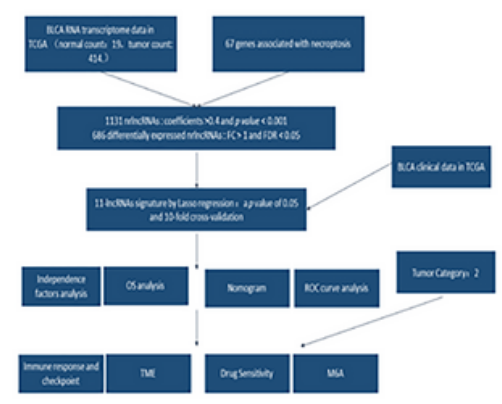

E

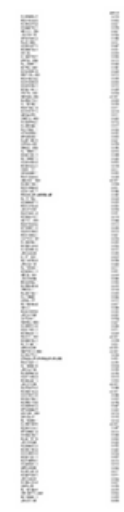

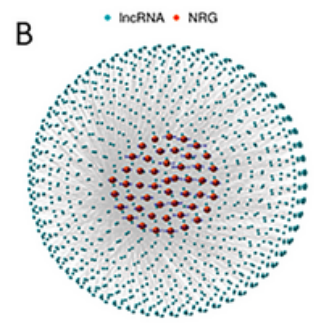

F

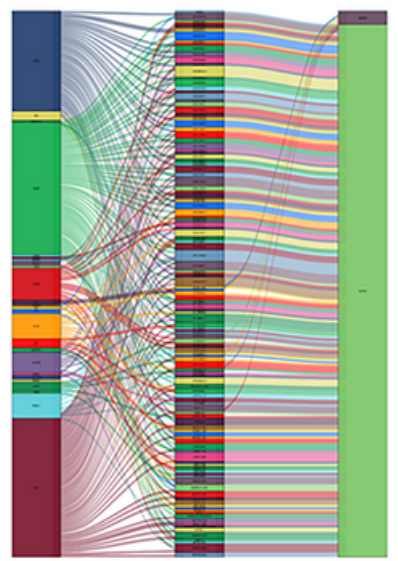

C

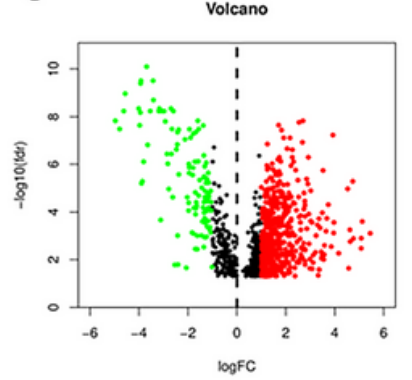

G

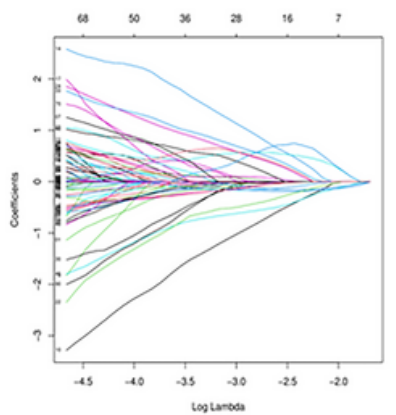

D

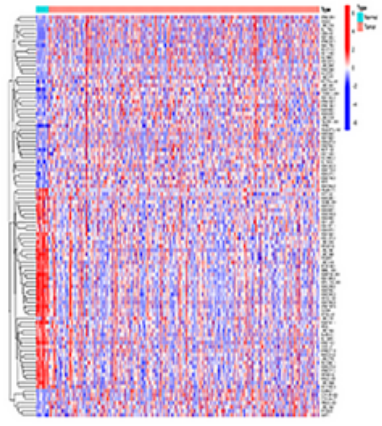

$\mathrm{H}$

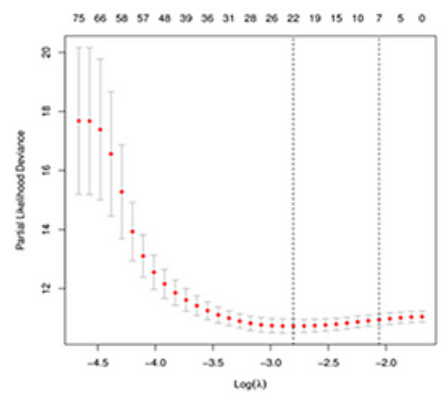

Figure 1

(A) Study workflow. (B)The interactive network between IncRNAs and NRGs. (C) Red Dots represent (log $F C>0)$ upregulated genes, whereas green dots $(\log F C<0)$ represent downregulated genes. (D) The expression profiles of 50 upregulated and downregulated nrlncRNAs each. (E) The prognostic nrlncRNAs $(p<0.05)$ extracted by univariate Cox regression analysis. $(\mathbf{F})$ The regulatory relationship between 
prognostic nrlncRNAs and NRGs was mostly positive, and only six nrlncRNAs were negative. (G) The coefficient profile of prognostic nrIncRNAs in the LASSO model. $(\mathrm{H})$ The partial likelihood deviance of the LASSO model shown by cross-validation.

A

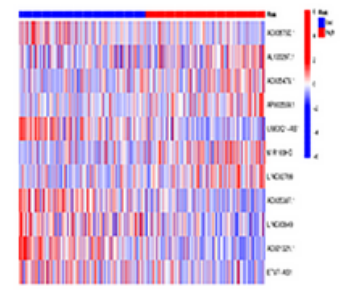

B

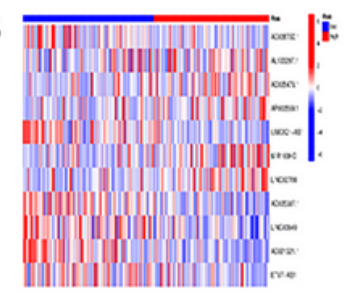

C

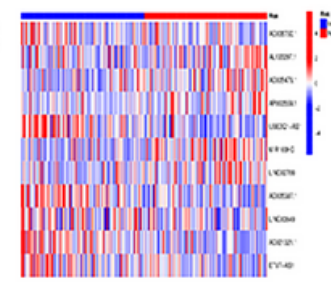

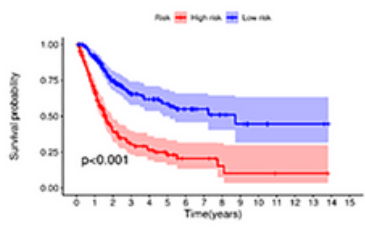

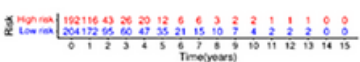

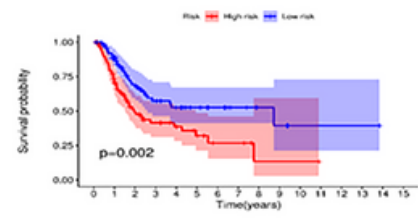

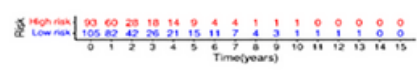

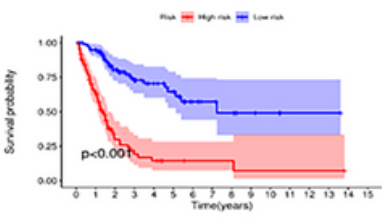

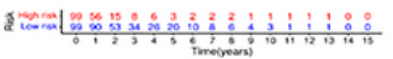
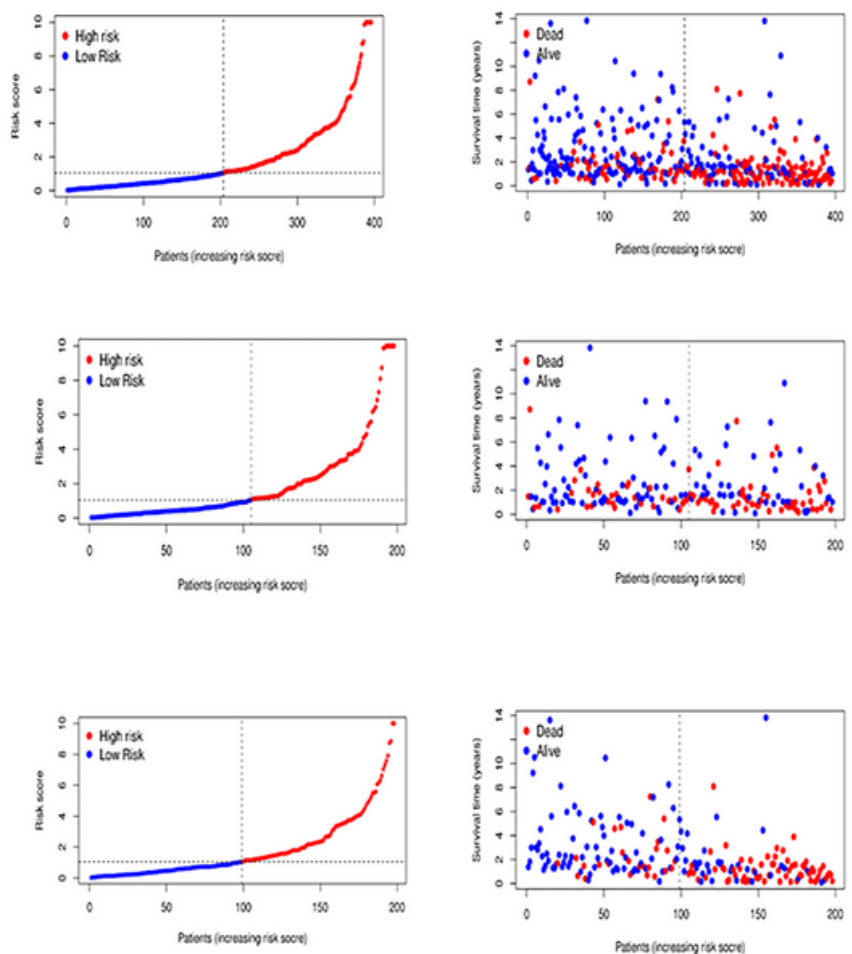

Figure 2

The exhibition of nrlncRNAs model based on 11-nrlncRNAs risk heat map, OS analysis $(p<0.05)$, risk score curve and survival status of the entire (A), test (B), and train (C) sets. 

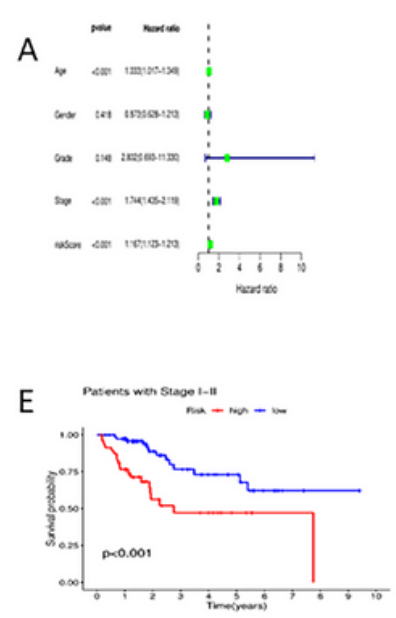
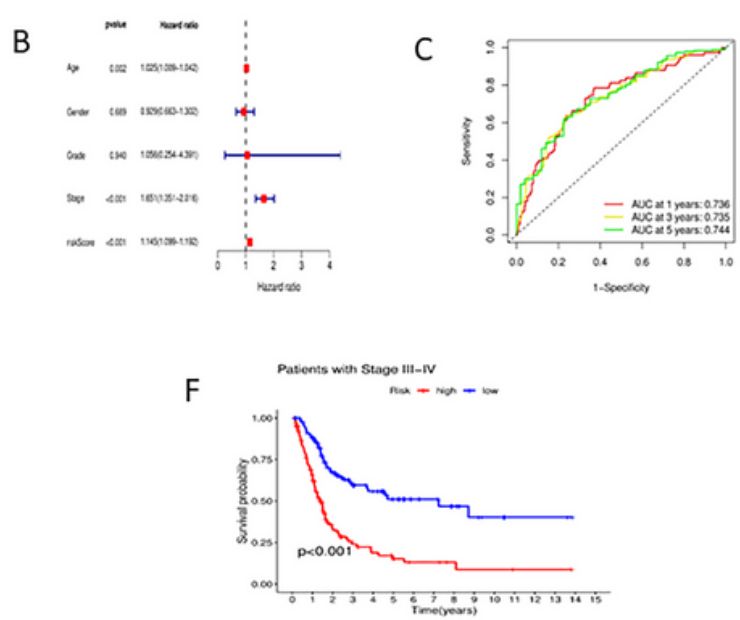

D

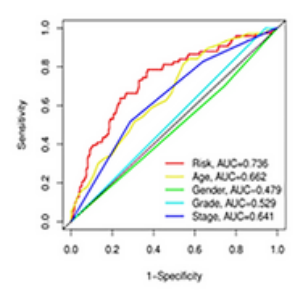

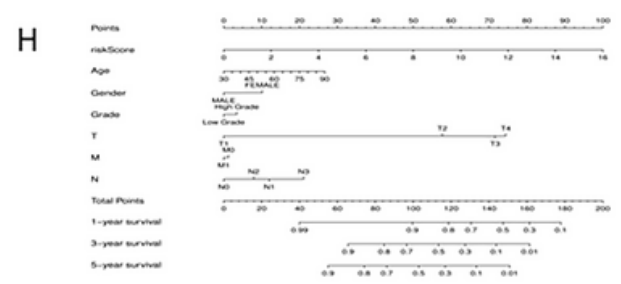
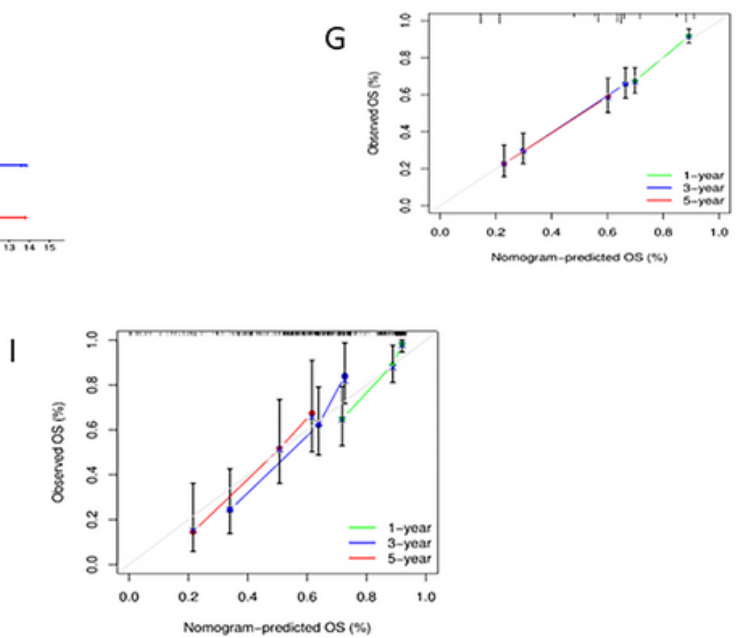

\section{Figure 3}

(A and B) Uni- and multivariate Cox analyses of risk score and clinical factors. Risk score $(p<0.001)$, age, and stage $(p<0.001)$ were statistically significant $(p<0.05)$. (C and D) The AUCs of $1-, 3-$, and 5 -years were greater than 0.7 . The AUC of risk was greater than those of other clinical factors. (E-G) In stages I/II and III/ IV groups, OS (survival probability) between high- and low-group had significant differences ( $p<$ 0.001). The 1-, 3-, and 5-year OS calibration curves are shown. ( $\mathrm{H}$ and $\mathrm{I})$ The nomogram and calibration curves that integrated the risk score, age, gender, and tumor TNM predicted the OS probability of the 1-, 3-, and 5-year. 
A

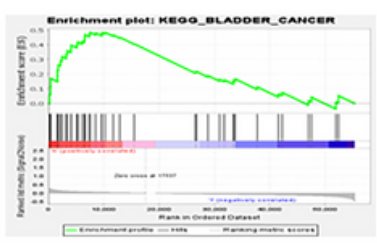

D
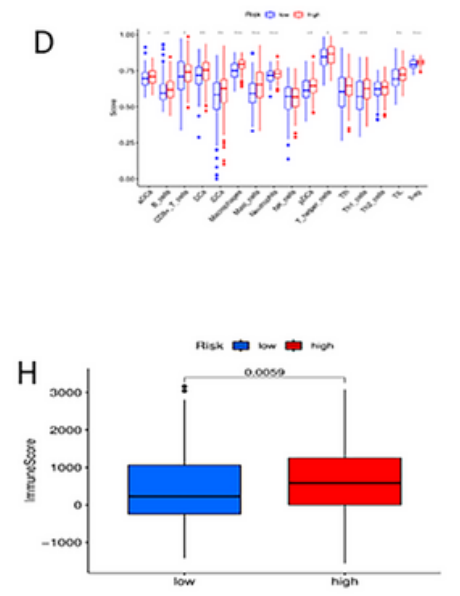

B

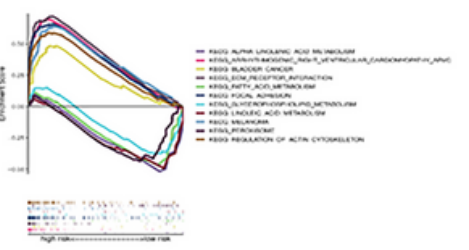

E

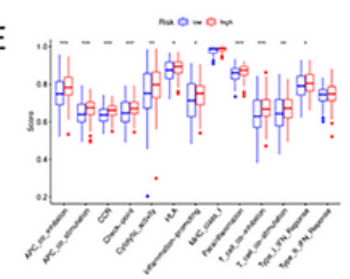

$\mathrm{F}$

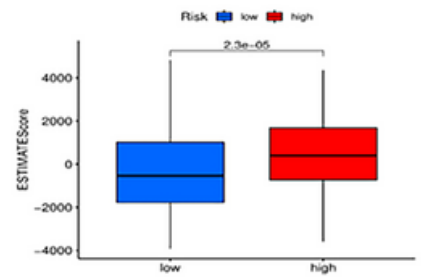

C
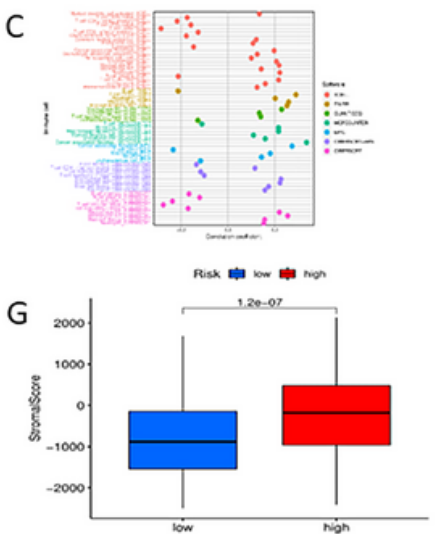
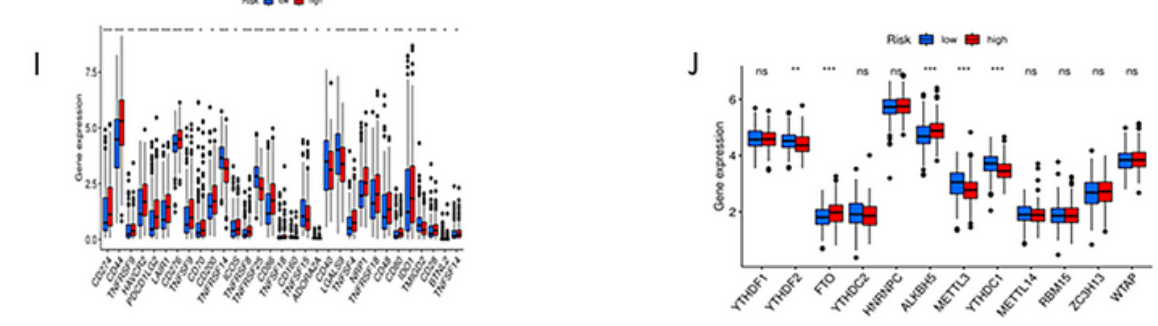

Figure 4

(A) The BLCA pathway GSEA was shown. (B) Multiple GSEA in risk groups. (C) The immune cells bubble of the relationship between the immune cells and the patient's risk score. (D) The SSGSEA differential expression analysis of immune cells in risk groups. (E) SSGSEA differential expression analysis of immune-related function scores in risk groups. (F-H) TME scores were compared between the high-and low-risk groups. (I) Immune checkpoint gene expression in risk groups. (J) M6A regulators gene expression in risk groups. 
A
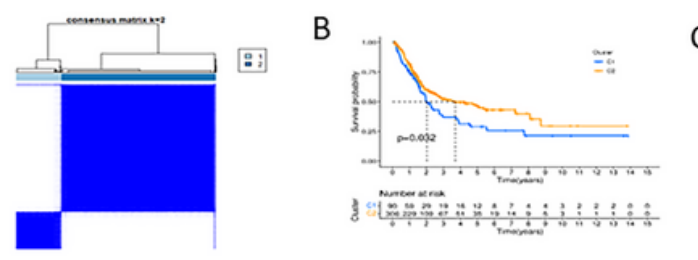

C

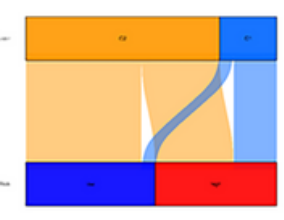

F

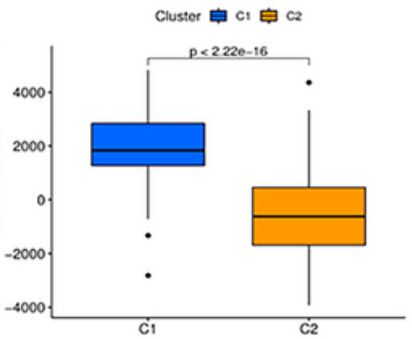

G

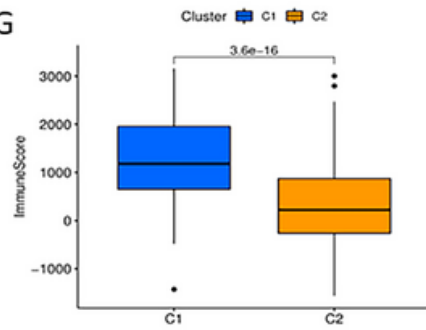

D

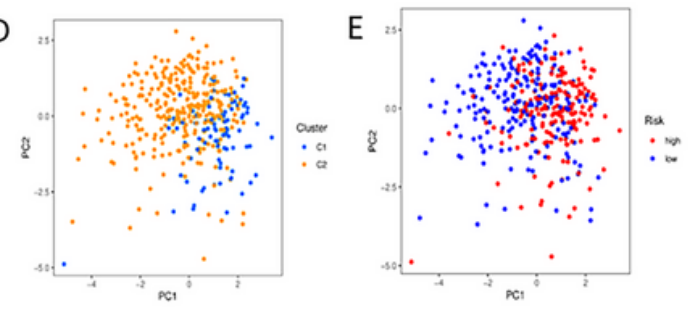

$\mathrm{H}$

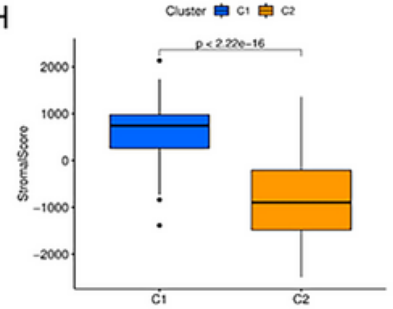

Cinsor 首

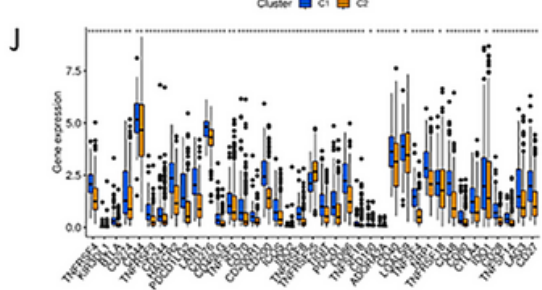

\section{Figure 5}

(A) The BLCA samples were divided into C1 and C2 clusters. (B) C1 and C2 clusters survival analysis. (C) The ggalluvial graph between risk groups and C1/C2 clusters. (D and E) PCA analysis in C1 and C2 clusters. (F-H) TME scores were compared between C1 and C2 clusters. (I) Heat map depicting different immune cells in the BLCA clusters. (J) Immune checkpoint gene expression in BLCA clusters.

\section{Supplementary Files}

This is a list of supplementary files associated with this preprint. Click to download.

- AppendixFigure1.jpg

- AppendixFigure2.jpg

- AppendixFigure3.jpg

- AppendixTable1.docx

- AppendixTable2.docx 